\title{
EcoSonic: Auditory Peripheral Monitoring of Fuel Consumption for Fuel-Efficient Driving ${ }^{1}$
}

\author{
Jan Hammerschmidt, Thomas Hermann \\ Ambient Intelligence Group, CITEC - Center of Excellence in Cognitive Interaction \\ Technology, Bielefeld University, Germany
}

\begin{abstract}
In this paper, we propose to make use of an auditory fuel efficiency display as means to support car drivers in adopting an energy-efficient driving style. We report on the development of the EcoSonic system as a platform for evaluating such displays and present five design approaches to guide their realization. In a study with 30 participants, we evaluated two prototype auditory displays against the baseline of visual-only eco-driving feedback in a within-subject study. Our selected designs are described in full detail. Key findings include a significantly reduced fuel consumption as well as lower engine speeds compared to the visual display. Furthermore, questionnaire analysis results confirm that the auditory conditions are less obtrusive and also seem to allow a more subconscious processing and comprehension of the provided information. Finally, we have found that the affectiveness of the display design seems to have a positive impact on its perceived helpfulness and the ability to absorb its information subconsciously.
\end{abstract}

Keywords: Auditory Interfaces, Eco-Feedback, Sustainable HCI, Behavior Change

\section{Introduction}

Eco-driving is the use of energy-efficient driving techniques in order to reduce the energy consumption when driving a car. Existing research highlights a range of opportunities and benefits that are associated with adopting

\footnotetext{
${ }^{1}$ http://dx.doi.org/10.1016/j.displa.2016.11.002

Preprint submitted to Displays (Special Issue on Realtime Sonification)

This manuscript version is made available under the CC-BY-NC-ND 4.0 license (http:

//creativecommons.org/licenses/by-nc-nd/4.0/)

November 2016
} 
such a driving style: First, as the energy consumption and pollution that is produced by both conventional and electric cars is one of today's major causes for greenhouse gas emissions [1], an energy-efficient driving style will have a major impact in terms of alleviating the negative effects of climate change [2]. Even more, contrary to current advances in building more and more efficient engines, eco-driving does not necessarily require to buy a new car, but can be applied to any existing vehicle. Furthermore, eco-driving is becoming even more important with hybrid and electronic cars, as the driving style has an even greater impact on energy consumption when compared to conventional combustion engines [3]. Finally, fuel-efficient driving generally also leads to a safer driving style [4] and can be done without a huge impact on trip time [5].

However, despite these advantages, the behavior change towards adopting these techniques poses to be a challenging one: Currently, there exist a number of visual fuel efficiency displays providing feedback on instantaneous or long-term fuel economy to support the driver in achieving a lower level of fuel consumption [6]. Observing these displays, however, requires a cognitive effort and can easily distract the user from the actual driving task and thus be detrimental to a safe steering of the car [7]. This is especially

problematic insofar as it is precisely in situations when drivers should keep their eyes on the road (e.g. when quickly accelerating or approaching a street crossing or traffic light) that the information from such a display becomes most relevant. Contrary to that, auditory displays have shown to be able to convey information in a less distracting way: Preliminary research on invehicle auditory interaction already hints at a significantly reduced impact on attention [8] as well as an improved effectiveness in terms of user performance [9], which is also supported by psychological research on multiple resources theory and dual task interference [10].

Although, taken together, this clearly supports the notion of an auditory fuel efficiency display, no evaluation of the efficacy and acceptance of such a system has been conducted before. The research presented in this paper fills this specific research gap and confirms preliminary findings concerning auditory in-vehicle interaction.

\section{Related Work}

While to our best knowledge, there is no research specifically studying the use of an auditory display for feedback on fuel consumption, the topic of eco-driving as well as the evaluation and comparison of visual fuel economy displays is well-covered in literature. Furthermore, more and more work is 
being done on the use of in-vehicle auditory displays in general [11] as well as for specific use-cases, e.g. collision warnings, skill acquisition, or in-car entertainment systems.

\subsection{Efficacy of eco-driving}

Although the findings for the precise amount of achievable reduction of fuel consumption vary to some degree, there already exist a number of studies evaluating the efficacy of eco-driving, which, taken together, clearly indicate a significant positive impact of eco-driving techniques.

Gonder et al., for example, evaluated the potential fuel savings that can be achieved by implementing different efficiency strategies while driving [12]. Using real-world data from trips collected with GPS devices and a vehicle model of a midsize car, they performed extensive simulations in order to assess the potential savings from specific behavior changes as well as the prevalence of inefficient (or: sub-optimal) driving. They conclude that for aggressively driven trips, the adoption of efficient driving behaviors can result in fuel savings of approximately $20 \%$ and even for more moderatelydriven trips, a 5\%-10\% reduction of fuel consumption is realistic.

In a recent study conducted by Sullman et al., a driving simulator was employed to train professional bus drivers in using eco-driving techniques [13]. By using consumption data provided by bus companies, they found out that six months after the training, fuel economy had improved by $16.9 \%$.

\subsection{Distraction by in-vehicle systems}

As the number of in-vehicle systems that are available to drivers is steadily increasing, there is a justified apprehension that these systems might cause considerable distraction and reduced driving performance, which in consequence could lead to an increased prevalence of traffic accidents. Several researchers have thus tried to quantify the effects of existing in-vehicle systems and find out the type of interaction that might best be used for those systems in order to alleviate the risk of distraction. For example, in a study with 23 participants, Lansdown et al. evaluated in which way distractions from in-vehicle information systems affect drivers [7]. The participants had to drive a test track in a driving simulator and were presented with simultaneous visual tasks, representing the interaction with additional in-vehicle systems. The researchers found out that those tasks led to significantly reduced headways and increased brake pressure. Additionally, they observed compensatory speed reductions and an increased self-reported 


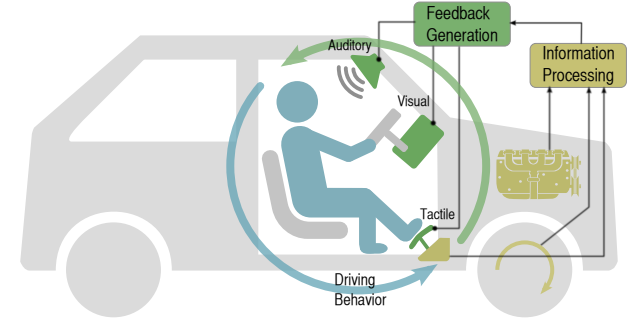

(a)

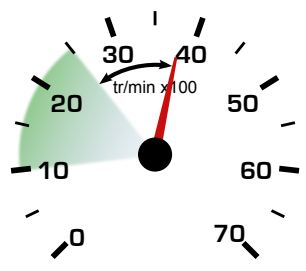

(b)

Figure 1: (a) Feedback loop for an eco-driving display [14]. The driver controls the (acceleration of the) car, which in turn controls the feedback display. The feedback can be generated in visual, auditory, or tactile ways, and gives supports in improving driving behavior. (b) Picture visualizing the basic idea of the metaphorical sonification display concept.

workload. Taken together, the authors see these results as evidence for a clear safety risk when using visual in-vehicle information systems.

On the other hand, there have been studies that hint at the advantages of employing the auditory channel: Jensen et al. conducted a study evaluating the impact of navigation systems on driving performance [8]. They compared different audio-visual output configurations for the system and found that a visual-only output not only led to a considerable amount of eye-glances, but also to a significant decrease in driving performance. While a user questionnaire indicated preference for an audio-visual configuration, the audio-only navigation system performed best in terms of those two aspects.

\subsection{Comparison of existing eco-feedback systems}

As there already exist a few (mostly visual) systems to provide feedback on eco-driving performance (also cp. Figure 1a), a number of studies try to assess and compare these systems in terms of efficacy and user acceptance. Tulusan and colleagues conducted a questionnaire survey and several semistructured interviews in order to learn more about which eco-feedback types might be preferred most by car drivers [15]. The authors learned that a comparison with an average consumption (e.g. of other drivers) seems to be beneficial for drivers to assess and better understand what could have been saved through a more ecological driving behavior. They also point out that most preferable were unobtrusive systems, which do not pose any additional workload (and frustration) for the drivers.

Jamson et al. compared the effectiveness of different configurations of either haptic, visual, or audio-visual eco-driving interfaces which provide 
feedback on a predetermined optimal accelerator pedal angle [16]. While the emphasis of the study was primarily on using a haptic accelerator pedal, the authors point out that for visual displays, the inclusion of complementary auditory feedback not only improved eco-driving performance, but also lowered visual distraction.

Finally, in a driving-simulator study, Hellier et al. evaluated how different levels of engine noise affect the driving style and perceived comfort [17]. They found out that low levels of engine noise led to increases in driving speed and more traffic violations. Surprisingly, the low-noise feedback conditions were also associated with a decrease in driver comfort. The authors conclude that auditory feedback plays a major part in the ability of a driver to make judgements and choices about speed.

\subsection{Auditory in-vehicle interaction}

While auditory interaction has long played a very minor role for use in automobiles, there recently has been considerable work towards using the auditory modality for in-vehicle interaction [11].

For example, Gable et al. studied the use of auditory cues for menu navigation in in-car entertainment systems. They evaluated eye tracking data, performance, workload, and user preferences in a recent study, where participants had to perform a list search on a cellphone, while doing a lane change task in a car simulator [18]. The list was presented either with no sound or one of five combinations of text-to-speech (TTS) audio, spearcons (i.e. algorithmically compressed speech), and spindex (an auditory cue based on the pronunciation of the first letter of a menu item). The authors discovered that the spindex+TTS cue not only allowed a significantly longer fixation time on the driving task than the visual-only condition, but was also associated with a decreased lane deviation.

In a different context, Powell and Lumsden developed and evaluated an auditory display to support motor sport drivers in improving their racing skills [19]. Based on a novel target matching design, the drivers were provided with tonal feedback on the lateral G-force on the one ear and with the target G-force (representing the limit of the car) on the other ear. Evaluation of the design showed promising first results, with the greatest efficacy of the system, when learning new tracks or familiarizing with new cars. Responses from the study participants also indicated a positive influence on the drivers' confidence.

Finally, Välstfjäll explored the design of in-vehicle auditory displays based on emotional reactions [20]. He conducted a study with 30 participants in a simulator environment with several more or less imminent colli- 
sion scenarios and evaluating the effect of auditory icons (i.e. naturally occurring, metaphorical used sounds) in comparison to abstract earcon sounds as well as testing 3D (spatial) sounds against monophonic sounds. In the experiment, auditory icons were found to cause a more intense emotional response for the participants as well as provoking faster response times (i.e. a quicker brake reaction) than the earcons. Similarly, spatialized sounds were perceived to be more activating and urgent, especially in a lateral warning situation, like a lane change scenario. Välstfjäll points out that the results clearly support the notion of emotion and behavior being tightly interconnected, suggesting that the emotional response should be a main aspect to be considered when designing auditory displays for inducing behavior change in the car.

In conclusion, this extensive review of literature shows not only the high potential of measures to support eco-driving, but also the clear benefits of using the auditory modality for doing so.

\section{General design approaches}

While current scientific knowledge supports the notion of an auditory fuel economy display, the specific design of such a system is something that clearly needs further research. As the possibilities for the actual realization are quite diverse, we have decided to first structure the design space: Following [14], we have developed five general design approaches to guide the development process of an auditory fuel economy system, which will be discussed below. Note that these approaches are not mutually exclusive, but could be combined to obtain a comprehensive display design.

We see the first and most straightforward approach to designing a fuel economy system as being the direct sonification of fuel consumption. Conceptually, this approach could be seen as a direct translation of existing visual fuel efficiency displays to the auditory domain. However, there are a multitude of design questions which need to be considered and which decide if the display can actually convey the intended information and if it will be accepted by potential users. For example, we must decide, if the display should be a continuous sonification (i.e. one that allows the driver to assess the fuel consumption at all times) or an event-based one, which is only emergent for a specific incident or situation, e.g. after consuming a specific amount of fuel, when the driver manages to achieve a comparably good fuel efficiency, or, on the other hand, when consumption is unusually high. For a continuous sonification, the main question is how the data is 
mapped onto the characteristics of a sound, e.g. if pitch, brightness and/or loudness should be modified based on the current fuel consumption. As unobtrusiveness seems to be a major factor for user acceptance [15], a crucial question is also how to achieve this design quality. One possibility would be to use the concept of blended sonification, which advocates the use and modification of already existing sounds [21]. In the context of a car, we could, for example, modify the sound of the engine in an appropriate way or subliminally modify the acoustic attributes of the music coming from the radio.

Secondly, a sonification of secondary parameters could be a promising way to support drivers in attaining a fuel efficient driving style. More specifically, this includes all aspects of driving that are known to affect the fuel consumption of a car. An aggressive driving style, for example, is characterized by phases of quick accelerations followed by unnecessarily hard (or too frequent) braking. An auditory fuel efficiency system could create an awareness for the negative effects of these behavior patterns. Another secondary parameter would be the number of revolutions of the engine: Just keeping those low can lead to a smoother and more fuel-efficient driving style.

The third design approach suggests using gamification as a conceptual framework to keep an auditory display engaging. The rationale behind this is the problem that the motivation to reduce one's fuel consumption might initially be quite high, but can also easily diminish over time. Gamification has shown to be able to motivate people over a longer period of time, most prominently for sports or health applications, but also, for example, for domestic energy engagement [22]. More specifically, this could mean a shift from conveying the fuel consumption in a "neutral" way towards a display that emphasizes the progress the driver makes towards a fuel-efficient driving style, e.g. by using virtual rewards, or introducing a way for social comparison.

A further design approach is to sonify advanced support information. This includes all aspects of the environment that have an influence on how the car can be driven, e.g. how far away an upcoming stop sign is, how much time a red traffic light will take to switch to green, or how fast the vehicle ahead is driving. Since this information usually becomes most relevant in safety-critical situations, an auditory display would have the tremendous advantage of not diverting the driver's attention away from the street.

Finally, supporting specific qualities of driving could be a very subliminal way to induce a more fuel economic driving style. With this approach, we are more interested in the psychological effects of a specific sound. For example, based on the hypothesis that there is a certain desire for fast driving, the 
sound design of a car could help convey this impression, e.g. by modifying the engine sound towards a more "sportive" feeling. In consequence, this should reduce the need to actually drive this way.

\section{Sonification Designs}

Based on the approaches discussed in the previous section, we have developed a range of prototype auditory displays, two of which are discussed here in more detail and have been evaluated in the study described in the following sections. Although the design approaches can guide the development of quite elaborate support systems, we have decided to first concentrate on relatively basic designs based on the first two approaches, as those are more comparable to the existing visual counterparts.

\subsection{Continuous sonification of fuel consumption}

Our first prototype auditory display is based on the approach of directly sonifying the fuel consumption (also cp. [14]). The basic idea of this design is to map the instantaneous, relative consumption (i.e. liters per $100 \mathrm{~km}$ ) to the frequency of a bandpass filter that is applied to a noise signal, resulting in a high-resolution representation of the input data, which, at the same time, should be rather unobtrusive for the driver. As the differentiation between levels of fuel consumption can be expected to be most important at the lower range of consumption, we mapped the input data logarithmically to the filter frequency. Furthermore, since the perceived loudness of the output signal obviously depends on its center frequency, we applied a basic psychoacoustic amplitude compensation to the generated sound. Finally, the output level is adjusted based on the loudness of the engine sound, i.e. a louder engine sound also leads to an increase of the output level of the sonification.

\subsection{Metaphorical sonification to support low-rpm driving}

The second prototype display (in more detail described in [23]) provides information about a secondary parameter to the driver: It encodes the instantaneous revolutions per minute of the engine through an event-based sonification. More specifically, a range of optimal rpm is defined based on the engine parameters, the selected gear, and the current slope of the street. When the user is driving at an engine speed that is higher than the upper threshold of this range, a continuous slurping sound is played back, which metaphorically represents the increased amount of fuel that is currently consumed (also cp. Figure 1b). Depending on how much the threshold is exceeded, the loudness of the auditory icon is increased. As the differentiation 
of the amount of this exceeding is obviously most important in the range directly following the rpm threshold, the loudness of the sound is increased logarithmically. Similar to the continuous sonification, we also compensate for the current loudness of the engine sound.

\section{The EcoSonic System}

In order to explore and assess the possibilities of auditory displays supporting fuel-efficient driving, we have developed the EcoSonic system for reproducible basic research. At its core, the system consists of a driving simulator with a precise model of a car and its engine. The simulator runs on an ordinary computer and can be operated with a steering wheel as well as two pedals for throttle control and braking. Gear selection is done with two buttons located on the steering wheel. To assess the effect of a specific auditory fuel economy display, a third component of the system logs the user's actively-made input to the simulator such as the pedal positions and selected gear as well as several simulated parameters, e.g. the car's instantaneous fuel consumption. Additionally, the system captures gaze information with the help of an eye tracker that is attached to the simulator's main display.

\subsection{The driving simulator}

The driving simulator can be considered the central component of the EcoSonic System. It not only includes a detailed model of the engine and the car to simulate a physically correct driving behavior, but also calculates a realistic instantaneous and accumulated fuel consumption.

Via an integrated $\mathrm{OSC}^{2}$ interface, the engine noise as well as individual auditory fuel economy display concepts can be controlled and played back through an external sound synthesis program like Supercollider ${ }^{3}$ or $\mathrm{Pyo}^{4}$.

Finally, a track editor can be used to design individual test routes for studying specific aspects of driving.

\footnotetext{
${ }^{2}$ OSC: Open Sound Control (http://opensoundcontrol.org)

${ }^{3}$ Supercollider: A real-time audio synthesis language (http://supercollider.github.io/)

${ }^{4}$ Pyo: Python module for digital signal processing (http://ajaxsoundstudio.com/software/pyo/)
} 


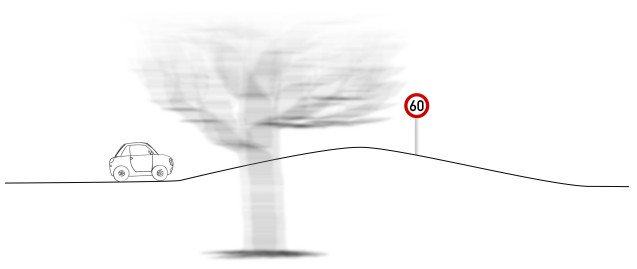

(a)

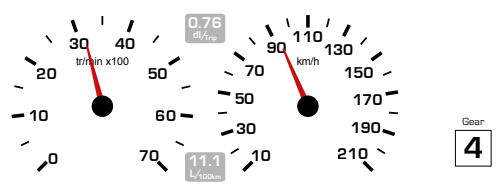

(b)

Figure 2: (a) Screenshot of the driving simulator. Due to the motion blur of the tree (which is rather near to the virtual camera), even in a still picture the movement of the car is perceivable. Also, the height profile as well as approaching traffic signs are clearly visible. (b) The dashboard of the car, which is displayed beneath the track view: The main elements are a display for the revolutions per minute on the left and a speedometer on the right. The instantaneous and accumulated fuel consumption are displayed in-between. On the right, there is an indicator for the currently selected gear.

\subsubsection{Graphical user interface}

One of the first choices that had to be made when developing the simulator was how to visually convey the actual process of driving. As for our usage scenario, the important aspect of driving is the acceleration behavior (i.e. not the steering of the car), we decided to implement a side-view perspective for graphically representing the driving scenario. This also has the advantage that we are better able to reproduce the scenario of a person driving a track on a regular basis: In this case, the details of the route are relatively well known to this person, which is reflected by the high visibility range of the side-view perspective.

Taken as a whole, the graphical user interface of the simulator consists of a track view and a view of the dashboard. The track view (Figure 2a) uses basically only four elements to visually display the driving scenario: A simple line represents the street, which, at the same time, can be seen as the height profile of the track. The street moves beneath the car, which is always at the left side of the screen, resulting in a constant visibility range. In order to give the user a sense of the speed of the car, several trees move in a virtual plane very near the camera, realizing a parallax scrolling effect. Depending on the car's speed, the images of the trees are filtered with a certain amount of motion blur, which adds to the impression of speed. Finally, there are several types of street signs (e.g. stop and speed signs) as well as traffic lights that can be placed along the track to model the characteristics of a specific route.

The dashboard consists of the typical elements that can be found in most cars currently in use, i.e. a speedometer and a display for the revolutions per 
minute of the engine (Figure 2b). Additionally, the instantaneous as well as the accumulated fuel consumption are displayed in a textual representation. As for our study most of the participants could be expected to be German, we used the (in Europe more commonly used) L/100kmh as a unit for the instantaneous consumption. However, this is easily switchable to other units, like for example MPG (miles per gallon), which is more prevalent in Englishspeaking countries. Lastly, an indicator for the currently selected gear is displayed next to the dashboard.

This part of the user interface can either be displayed below the track view on the same screen or on a separate display, which then can be positioned independently, e.g. to simulate two viewing planes.

\subsubsection{Internal simulation}

The second important component of the driving simulator consists in the internal simulation of the car itself. This includes the computation of the car's movement depending on the user's input (e.g. throttle control and gear selection) and environmental factors (e.g. slope of the street). Of particular importance for the EcoSonic project is additionally the calculation of fuel consumption, i.e. how much fuel is consumed depending on the torque and revolutions per minute of the engine.

Engine model. The basic function of a car engine is to produce a certain rotary force (i.e. torque $\tau$ ), which, through the gearbox, drives the wheels of the car, thus moving the vehicle. The power output of the engine then is $P=\tau \cdot \omega$, where omega is the angular velocity.

For an internal combustion engine, the torque output is considered usable only over a limited range of rotational speeds $\omega$, typically between $1000 \mathrm{rpm}$ and $6000 \mathrm{rpm}$. But also within this range, output torque varies not only based on user-adjusted throttle, but also depending on the rotational speed, and reaches its maximum only at a specific (range of) $\omega$. In order to freely model the behavior of the engine, the simulator uses a torque map, which makes it possible to define several torque responses for different throttle positions. The exact torque output is then calculated by linearly interpolating between the previously defined torque response curves.

Fuel consumption. A crucial aspect for the evaluation of an auditory fuel economy display in a simulated environment is of course the fuel consumption of the engine. The consumption primarily depends on the engine's power output $P$, i.e. the fuel consumption $C_{f}$ would be $C_{f}=c_{E} \cdot P$, where $c_{E}$ is a engine-dependent coefficient. 


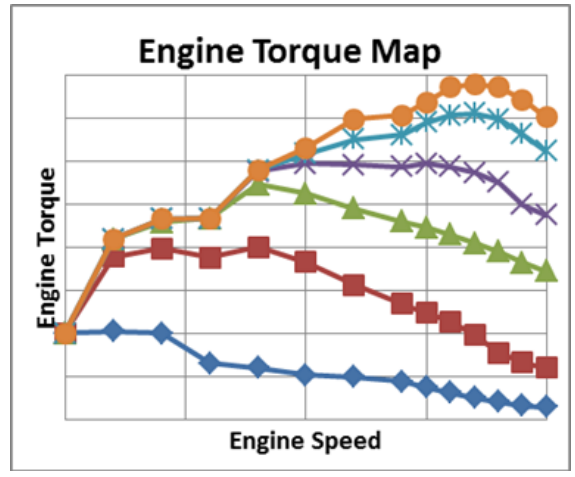

(a)

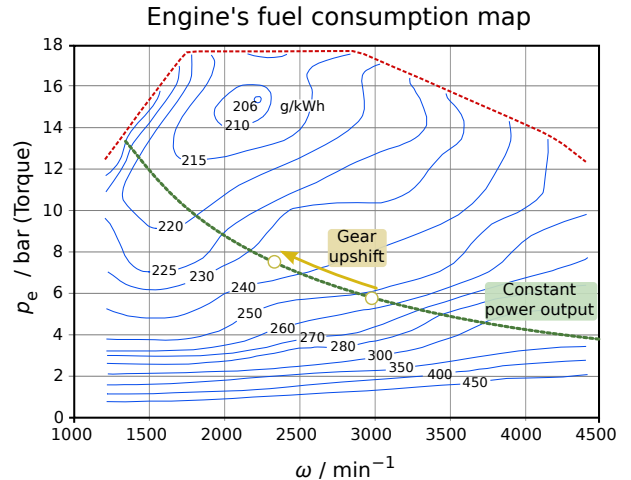

(b)

Figure 3: (a) A typical torque map of an engine [24]. Each of the differently colored curves represents a specific throttle position. Contrary to what one could expect, the output torque varies significantly even for the same throttle position, depending on the engine's speed $\omega$. (b) Exemplary representation of a fuel consumption map depending on number of revolutions and torque [23]. The green line illustrates a typical level of constant power output, i.e. moving on this line does not change the effective thrust of the engine. We can easily see that altering rpm by shifting gears has a significant influence on the fuel consumption of the engine. (Original picture from [25])

It turns out, however, that $c_{E}$ also depends on the current speed and torque of the engine, so that $C_{f}=c_{E}(\tau, \omega) \cdot P$, with $c_{E}(\tau, \omega)$ being an enginespecific function, which can be characterized by a fuel consumption map as shown in Figure 3b, and is also used internally by the driving simulator in order to precisely model the engine's consumption characteristics.

\subsubsection{Traffic violations and attention task}

In addition to the track itself (i.e. the elevation of the street), the driving environment primarily consists of street signs such as speed limits, stop signs, or traffic lights. Each of these signs introduces a specific rule for the driver, which can, of course, be violated. Besides informing the user about such traffic violation, e.g. driving too fast or ignoring a stop sign, these incidents are also logged by the system, as they are an indication for how much attention the user is able to devote to the street.

Furthermore, contrary to conducting real world studies, where distractions and needed attention are basically unpredictable, having a completely simulated environment also enables us to introduce a fully controlled attention task, which is the same for each study participant. As the side-view scenario does not require the user to steer, we decided to include an addi- 


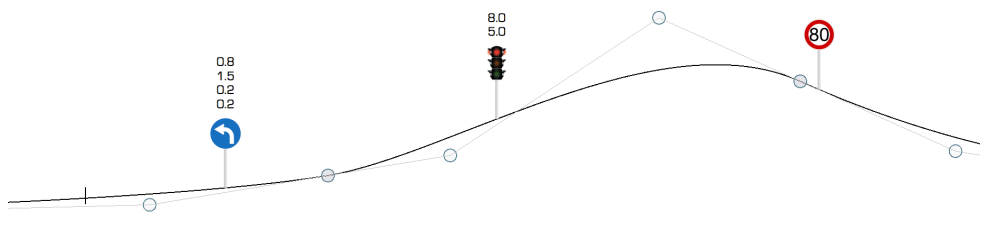

(a)

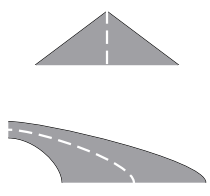

(b)

Figure 4: (a) Screenshot of the track editor of the EcoSonic System. The gray and white points represent the end and control points of the individual Bézier curves. The turn-left sign represents an attention task, where the iconic road is making a gradual left turn. The trigger distance of the traffic light is indicated by the vertical line on the left. (b) Two images of the iconic road, which is displayed above the track view as part of the attention task. In case of the upper image, the user would have to steer to the left.

tional steering task, which requires the user to react to simulated curves: At the center of the track view, an iconic representation of a street is displayed, which can gradually turn into a curve (Figure $4 b$ ).

\subsubsection{Track editor}

In order to make it possible to quickly design a specific driving scenario, the simulator includes a dedicated track editor, which allows the modification of the street's elevation profile as a cubic Bézier spline, where the two control points next to a curve's endpoint are kept collinear in order to maintain C1 continuity (cp. Figure 4a).

Additionally, street signs and traffic lights as well as attention tasks can be placed along the road. Traffic lights by default start showing a red light and are triggered to switch to green when the user is approaching it at a specifiable distance. The exact moment of the switch is then determined either by an explicitly specified or a random delay time. In order to keep the results as comparable as possible, only traffic lights with an explicitly specified delay time were used in the conducted study.

For the attention tasks, the amount of added curvature as well as the duration of the curve (i.e. how long the curvature will be modified) can be specified.

\subsubsection{OSC communication}

As the primary use of the driving simulator is to evaluate auditory fuel economy displays, it is mandatory to have an interface that allows for the design of prototype sonifications with the help of an external sound synthesis 


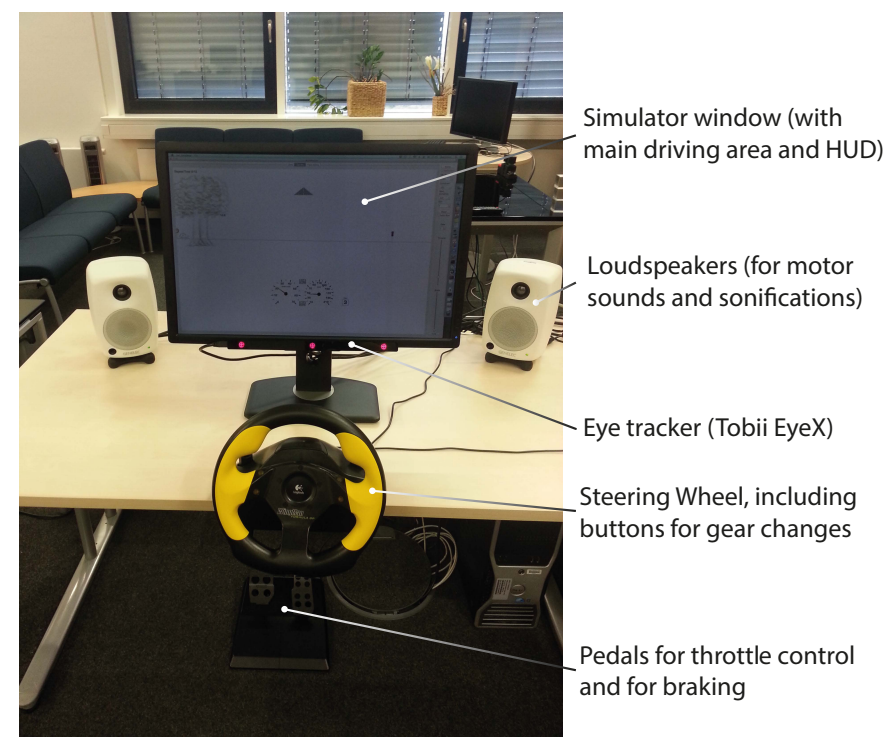

Figure 5: Hardware setup of the EcoSonic system.

program. Since the majority of those programs are able to communicate via the specialized Open Sound Control (OSC) protocol, the simulator offers an OSC-based interface that provides the instantaneous fuel consumption as well as regular messages indicating the expenditure of a specific amount of fuel.

Additionally, the interface is used for the synthesis of the engine sound and for that provides real-time updates on engine speed and the currently provided torque.

\subsection{Hardware setup}

The required hardware for the EcoSonic System basically consists of only 6 components and is therefore easily installed. For our setup, a Mac Pro was running the driving simulator, displaying the user interface on an 24-inch monitor. Attached to the monitor was an eye scanner to capture real-time gaze data of the user. For our purposes, we decided to use an Tobii EyeX tracker $^{5}$, as the accuracy and response time are sufficient and the calibration procedure is quite fast. As this eye tracker can only track gaze information for one display, the dashboard was not shown on a separate monitor, but

\footnotetext{
${ }^{5}$ http://www.tobii.com/en/eye-experience/
} 
below the track view on the main screen. We placed a pair of loudspeakers ${ }^{6}$ next to the monitor, as can be seen in Figure 5. In front of the display, a steering wheel was attached to the table and pedals for throttle control and braking placed on the floor ${ }^{7}$. Users were sitting in front of the setup so that they could comfortably operate the steering wheel and were approximately $60 \mathrm{~cm}$ away from the monitor for the best eye tracking performance.

\section{Study Design}

With the help of the EcoSonic system, we conducted a study to evaluate the prototype auditory fuel efficiency displays discussed in Section 4.

To reduce the number of necessary participants, we employed a withinsubject design. For each condition, the participants had to drive the same test track four times in order for them to familiarize with the respective display. Controlling for ordering effects between the different conditions, we employed a counterbalanced measures design, where all possible sequences of conditions (i.e. all 6 permutations) were evenly distributed over the study participants. In total, 30 people took part in the experiment.

At the beginning, each participant was given a short introduction, explaining the purpose of the experiment and a short questionnaire dealing with general questions about personal attitudes and preferences as well as previous experiences. Subsequently, the EcoSonic System, including the various elements of the driving simulator, were explained and the participants were told what they had to do during the experiment. Specifically, the participants were given 4 tasks:

1) They should follow the common (universally known) traffic rules: More precisely, they were told to adhere to the respective speed limits, to stop in front of a stop sign and not to drive through a red light. If they committed one of these traffic violations, they were notified about this incidence by a sound of a camera taking a picture in conjunction with an implied flash (the screen would go white and quickly fade back to normal), in order to give the impression of being caught speeding. Additionally, a short text would say, which traffic rule had been violated.

2) Also, they were told not to obstruct the traffic, i.e. they should not drive too slowly, depending on the current speed limit. If they did, this was

\footnotetext{
${ }^{6}$ Gendelec $8020 \mathrm{C}$

${ }^{7}$ For our setup, we used a consumer-grade steering wheel (Logitech Wingman Formula GP), which also has pedals included.
} 
indicated by the honking sound of a car, combined with an appropriate text message.

3) The participants were told to "keep on the street". This referred to the attention task, discussed in Section 5.1.3. More specifically, they were told to keep the curve-indicator in a neutral position (cp. Figure 4b).

4) Finally, the participants were instructed to keep the fuel consumption as low as possible. It should be noted, however, that they were not told how exactly they should do this.

Before the actual experiment, each participant was given the possibility to get used to the driving simulator on a separate short test track, which included all important elements (e.g. traffic signs) in a compact manner.

Then, for each condition, the respective fuel efficiency display was shortly explained, after which they could independently conduct the four runs. Afterwards, individual questionnaires dealing with specific questions about the particular display were handed out.

At the end of the experiment, the participants completed a final questionnaire with comparative questions and asking for feedback about fuel efficiency displays in general and the experiment as a whole. Altogether, the study lasted around 50 minutes for each participant, primarily depending on the time needed to complete the questionnaires.

\subsection{Goals and Hypotheses}

The primary goal of the experiment was to compare the two designs for an auditory fuel efficiency display to the well-established visual display found in most cars these days. More specifically, we wanted to find out, how they affect the driving behavior and fuel consumption, if there are any differences in terms of how much they distract the driver from the primary driving task, and how they are perceived by potential users.

Based on previous work [20], we hypothesized that the metaphorical sonification (MS) would be perceived as more affective $(\mathrm{H} 1.1)$ and, in consequence, more persuasive (H1.2). Furthermore, as the auditory displays effectively add functionality to the driving experience, we expected them to be assessed as more helpful than the visual display alone (H1.3). On the other hand, based on previous experience with auditory displays, we also saw the danger of them being slightly annoying, i.e. less likely to be accepted as a part of an in-vehicle interface (H1.4).

In terms of quantifiable influence on the driving behavior, we firstly expected the fuel consumption to decrease over the course of the experiment due to participants familiarizing with the test track and the driving simulator $(\mathrm{H} 2.1)$. Furthermore, we expected that the consumption would be lower 
when participants are supported by one of the auditory fuel efficiency displays (H2.2). Specifically for the continuous sonification (CS), we expected users to better be able to estimate the instantaneous consumption $(\mathrm{H} 2.3)$ and to look less often at the visual display (H2.4). The metaphorical sonification was expected to support drivers in employing lower engine speeds in comparison to the other conditions $(\mathrm{H} 2.5)$ and to cause less glances to the rpm display $(\mathrm{H} 2.6)$.

Following [17, 9], we hypothesized that participants would be able to comprehend the information provided by the auditory fuel efficiency displays on a more subconscious level (H3.1). Based on this hypothesis, we anticipated the displays to be less distracting, i.e. more unobtrusive (H3.2), and in consequence to alleviate mental overload while driving (H3.3).

Finally, these attributes were expected to provoke fewer traffic violations (H3.4) and to allow the drivers to focus more on the street (H3.5), thus enabling them to better "keep on the road" for the steering task (H3.6).

\section{Results: Questionnaires}

Based on the previously defined hypotheses (Section 6.1), we designed several Likert-type scales consisting of up to 4 questions, which had to be answered on a 7-point Likert scale either after each condition or at the end of the experiment as a comparative question. In the subsequent analysis, we considered composite scales with a Cronbach's alpha $>0.7$ in order to only allow question groups that had been consistently answered. Furthermore, a Friedman test was used to determine if there are any significant differences between the three conditions $(p<0.05)$. Then, for comparison between the individual conditions we used the Wilcoxon signed rank test to check if the differences between two scales are statistically significant. Finally, to compensate for multiple testing, the Benjamini-Hochberg False Discovery Rate method was used to adjust the resulting p-values. When testing for correlations, we combined the $r$-value of the Spearman rank-order correlation coefficient with the $p$-value of Kendall's Tau, as it is more accurate for smaller sample sizes.

The majority of study participants stated to have very well understood both the visual fuel efficiency display (VD) and the metaphorical sonification (MS) (median $\mu_{\frac{1}{2}}=7$ for both conditions). The continuous sonification (CS) was also well understood $\left(\mu_{\frac{1}{2}}=6\right)$, although the data shows a significant difference to the two other conditions $(p<0.02)$. 


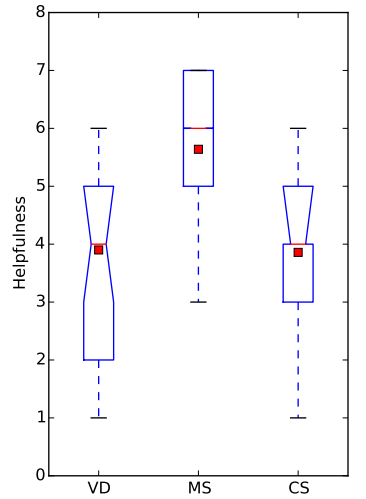

(a)

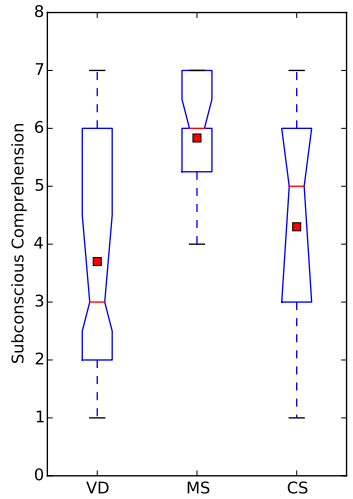

(b)

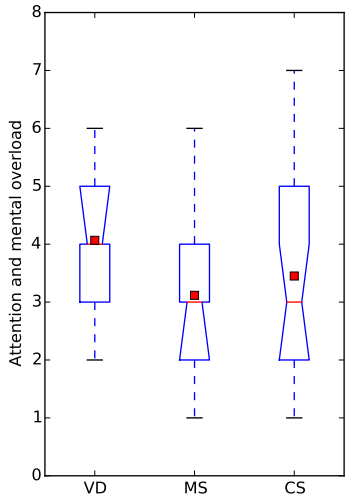

(c)

Figure 6: Comparisons of the different modes for some of the items of the questionnaire. The notches represent the $95 \%$ confidence intervals of the median.

When asked about being able to estimate the current level of fuel consumption, the two auditory conditions were rated only insignificantly better $\left(\mu_{\frac{1}{2}}=5\right)$ than the $\operatorname{VD}\left(\mu_{\frac{1}{2}}=4.5\right)$, with Friedman $p=0.24$, thus no statement can be made for $\mathrm{H} 2.3$. However, the participants assessed the MS as significantly more persuasive $\left(\mu_{\frac{1}{2}}=6\right)$ than the two other conditions (both $\mu_{\frac{1}{2}}=4$, with $p<0.01$ ), confirming H1.2. A very similar result can be seen for questions about the helpfulness of the three designs (Figure 6a), which partly verifies H1.3. This similarity between persuasiveness and helpfulness is also reflected in a strong correlation between these two variables $(r=0.87$, $p<0.01)$.

We also asked the participants to assess the unobtrusiveness of the different fuel efficiency displays, i.e. if they would be distracted by them while driving. Here, the CS $\left(\mu_{\frac{1}{2}}=5\right)$ was rated significantly better than the VD $\left(\mu_{\frac{1}{2}}=3.5\right.$, with $\left.p=0.02\right)$. The MS was rated even higher $\left(\mu_{\frac{1}{2}}=6\right)$, although the differences to the CS were not significant $(p=0.12)$. Similar results were obtained for the question if the participants had to concentrate on the fuel efficiency feedback or if they had been able to subconsciously absorb the presented information: Both CS and MS were rated significantly better $(p<0.01)$ than the VD (Figure 6b). Again, the similarity can also be seen when testing for correlation between the variables $(r=0.78, p<0.01)$. These findings confirm both H3.1 and H3.2. 
The differences in terms of unobtrusiveness and subconscious comprehension may explain, why the participants stated that they felt overwhelmed by the driving task and had difficulties to keep their attention on the street significantly more for the visual condition $\left(\mu_{\frac{1}{2}}=4\right)$ than for the auditory ones (both $\mu_{\frac{1}{2}}=3$, with $p \leq 0.03$ ) (Figure 6 c), which verifies H3.3.

Interestingly, when asked if the respective designs would be annoying while driving (i.e. how well they would be accepted), the participants rated the MS $\left(\mu_{\frac{1}{2}}=6\right)$ slightly better than the VD $\left(\mu_{\frac{1}{2}}=5\right)$ and the CS $\left(\mu_{\frac{1}{2}}=\right.$ 3.5). Although the differences between the conditions were not significant (Friedman $p>0.08$ ) and thus no statement can be made for $\mathrm{H} 1.4$, these results are clearly in favor of the design of the metaphorical sonification.

Finally, we asked the participants, how the different fuel efficiency displays appealed to them at an emotional level. While this was basically not the case for the $\operatorname{VD}\left(\mu_{\frac{1}{2}}=1\right)$, it was significantly more so for the MS ( $\mu_{\frac{1}{2}}=4, p<0.01$, confirming H1.1), but also for the CS $\left(\mu_{\frac{1}{2}}=2, p=0.01\right)$. Interestingly, the affectiveness of a display was positively correlated with its helpfulness $(r=0.3)$ and the ability to absorb its information subconsciously $(r=0.23)$. On the other hand, it also had a small impact on its understandability $(r=-0.27)$. Correlation to persuasiveness was surprisingly low $(r=0.21)$, albeit still significant $(p<0.01)$.

While it is not surprising that the understandability $(r=0.58)$ as well as the perceived helpfulness and unobtrusiveness (both $r=0.6$ ) of a fuel efficiency display were highly correlated with its acceptance, the possibility of subconscious comprehension had an even greater positive influence $(r=$ $0.67, p<0.01)$.

\section{Results: Measured data}

The second part of the evaluation deals with the data collected by the EcoSonic system, including data from the driving simulation as well as the eye tracker.

\subsection{Fuel consumption}

In Figure $7 \mathrm{a}$ we can see the total fuel consumption plotted against the time needed to complete the whole test track, which gives a rough impression on how these two variables depend on each other. Additionally, we performed a least squares fit of a linear function to this data. As could be expected, the participants that were faster consumed on average a little 


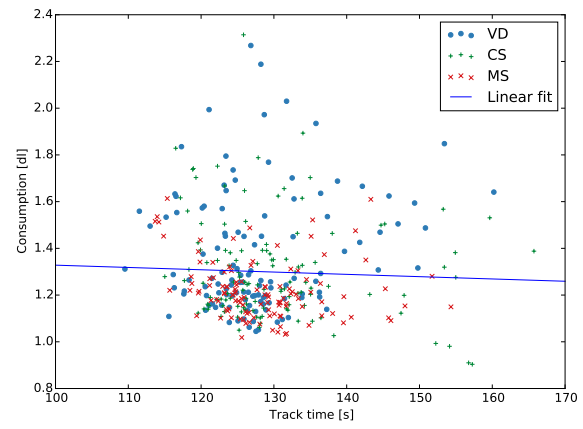

(a)

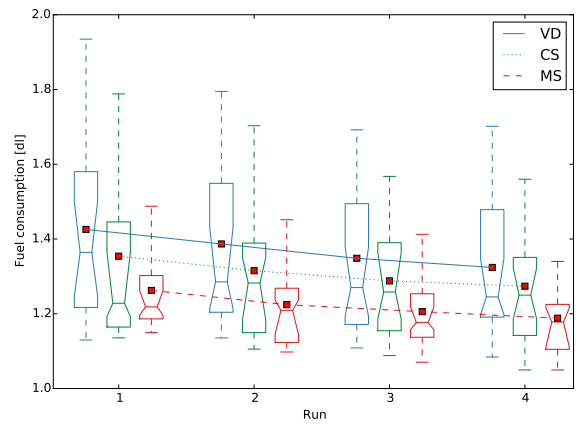

(b)

Figure 7: (a) Scatter plot showing the fuel consumption in dependence of the time the participants needed to complete the test track. Additionally, a least squares linear approximation of the data is overlayed. (b) A boxplot comparing how much fuel was consumed for the three conditions over the course of the four runs. The continuous lines connect the average values for each respective run.

more fuel than the ones taking their time. However, the dependence is surprisingly low (Pearson correlation coefficient: -0.04) and statistically not significant $(p=0.44)$. These findings are in line with previous work examining the potential to reduce fuel consumption without a significant impact on trip time [5].

Comparing the measures of fuel consumption over the course of the four runs, we can see that for each condition the participants consistently improved their fuel efficiency (Figure 7b), which is consistent with our hypothesis H2.1. Due to the high variance of the data, however, the differences between the first and the last run are significant only for the MS $(p=0.02)$.

Furthermore, the average fuel consumption differs between the three conditions (Friedman $p<0.01$ ): Confirming our $\mathrm{H} 2.2$, it is significantly higher for the visual condition $(1.37 \mathrm{dl} \pm 0.25)$ compared to the auditory ones $(p<0.01)$, but also lower for the MS $(1.22 \mathrm{dl} \pm 0.12)$ than for the CS $(1.30 \mathrm{dl} \pm 0.22)(p<0.05)$, establishing a clear order of $\mathrm{MS}<\mathrm{CS}<\mathrm{VD}$.

\subsection{Engine speed distribution}

Analyzing the participants' driving behavior, Figure 8a illustrates how much time during the test track the car was driven at a certain engine speed. We can clearly see that participants driving with the MS used the lowest engine speeds, which can be confirmed by statistically comparing the average engine speed over the whole track $(p<0.01$ when comparing the MS $(1767 \mathrm{rpm} \pm 307)$ to the VD $(1994 \mathrm{rpm} \pm 323)$ and CS $(1889 \mathrm{rpm} \pm 307))$. This 


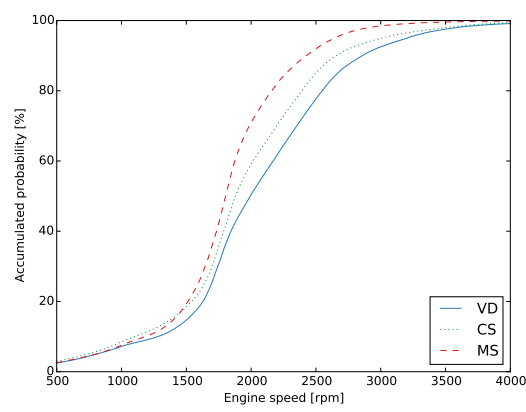

(a)

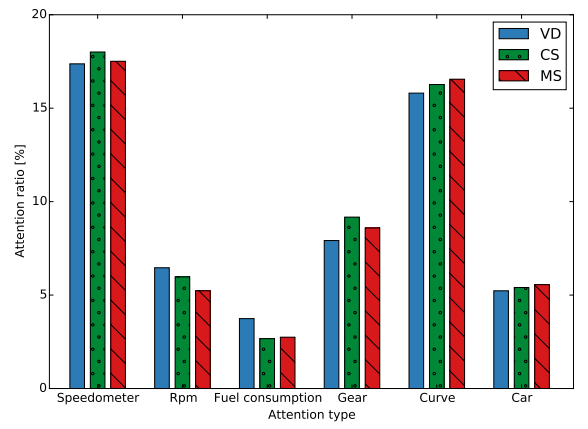

(b)

Figure 8: (a) Cumulative distribution function of the average engine speed distribution for the different conditions. (b) Attention ratios for a selection of points of interest.

finding confirms the effectiveness of the metaphorical sonification, which was specifically designed to help driving with lower rpm, and verifies our hypothesis H2.5. Furthermore, we can see that sonifying the fuel consumption (CS) enabled the drivers to reduce the average engine speed as well, which is especially remarkable considering that also for the visual condition, participants were given the task to drive as fuel-efficient as possible (cp. Section 6).

\subsection{Traffic violations and steering task}

On average, the participants committed 0.52 traffic violations for the whole test track, which can be considered quite few. Also, the differences between the individual runs were quite high $(S D=0.72)$, making it difficult to establish any statistical statements. Thus, while it could be observed that drivers supported by the CS committed marginally fewer traffic violations ( 0.44 vs 0.58 for the VD), we could not find any significant differences between the three conditions and therefore can not make any statements concerning hypothesis H3.4.

Similarly, data collected from the steering task contained a high variance that could not be attributed to a specific condition: As a measure for the level of distraction, we analyzed the instantaneous deviation from the neutral curve position $\delta^{s}$ and calculated the normalized integral $\delta_{r}^{s}=$ $\left(\int_{0}^{t_{\text {run }}}\left|\delta^{s}(t)\right| d t\right) / t_{\text {run }}$ for each run. Surprisingly, the differences of the average $\delta_{r}^{s}$ between the conditions are maximally 1\%, while having a high coefficient of variation $\left(c_{v}=0.58\right)$. Consequently, hypothesis H3.6 can not be confirmed. Considering that $-1<\delta^{s}<1$, the mean $\delta_{r}^{s}$ is rather high (0.24). With this data, we can only hypothesize that the participants paid 
less attention to this comparably new and unfamiliar task, regardless of the respective condition.

\subsection{Eye tracking data}

For analyzing gaze information that was provided by the eye tracker during the experiment (cp. Section 5.2), we first classified for each simulation step of the driving simulator, which part of the GUI the participant was looking at, based on a simple area matching. Then for each item, an attention ratio was calculated based on the accumulated time it was looked at in relation to the total time of a run. Invalid or unclassifiable data (i.e. gaze points outside the application window) were discarded. In Figure 8b, the average attention ratios for each condition are shown.

One first observation we can make is that participants looked at the speedometer more often than expected (i.e. $17.64 \%$ of the time), which might be attributed to difficulty in estimating their speed.

Furthermore, although the average attention ratio is comparably low, we can see that for both the MS $(2.75 \% \pm 2.84)$ and the CS $(2.67 \% \pm 2.44)$, participants looked less often at the fuel consumption display than for the VD $(3.74 \% \pm 3.29)$. The differences are significant ( $p \leq 0.01$ for both comparisons) and verify our hypothesis $\mathrm{H} 2.4$.

We can also see that the total time spent looking at the display for the revolutions per minute was lowest for the MS $(5.23 \% \pm 4.84)$ and significantly less than for the VD $(6.46 \% \pm 5.15)(p<0.01)$, which confirms our hypothesis H2.6. For the CS, it was not as low $(5.97 \% \pm 4.93)$, but still lower than for the VD $(p=0.03)$.

While for the auditory conditions the users seemed to have slightly more time to keep an eye on the curve indicator as well as the car itself, these differences are not significant, which prevents any statement on H3.5.

\section{Discussion}

An important factor for the integration of the information sources into behavior is the familiarization: Under all conditions, the fuel consumption decreases from run to run, indicating that users gain a successively better understanding of the parcours, how the controlled vehicle reacts on interaction, and how to drive in a fuel-economic way. We only provided 4 runs per condition, wherein we witness rather similar improvement curves as function of run number (see Figure 7b). Notably, the auditory conditions are already better from the onset (first run) and improve along a similar asymptotic pattern as under the visual condition. We do not have sufficient data to 
fully understand the mechanisms, yet one plausible explanation is that with the sonification the information is already correctly and timely conveyed so that it immediately helps users in saving fuel, and the subsequent improvements come from mastering the driving task successively well, independent from fuel consumption. This is also supported by the widely acknowledged multiple resource theory [10], which suggests that attending to a visual fuel consumption display can be expected to cause a significant interference with the visual task of car driving, while attending to a peripheral auditory display can be assumed to be relatively resource-free.

EcoSonic exemplifies a more general motif of using sounds as feedback for human actions: It emphasizes sound's potential to influence routines and habits. Routinization and the emergence of habits are processes that quite generally occur on repeated executions of tasks, normally (a) to optimize the required effort and (b) to relieve the user from a conscious and thus cognitive mode of processing information. Automatization simply means that establishing routines allows us to set cognitive capacity free for tasks and goals between the level of task execution. It happens virtually everywhere, e.g. to walk, to drive a bike, the way we eat or brush our teeth, or how we take a shower. Once the actual micro-management of action steps is sunken below the threshold of conscious attention, we may become detached from detailed reflection of how we do things and the repeated (unquestioned) execution strengthens the habits as learned. In order to change habits, we believe that instantaneous real-time feedback concerning a refined objective function is highly instrumental (in case of EcoSonic: the instantaneous fuel-consumption). The novel feedback - particularly if its meaning with respect to data is understood, and more so if the refinement of routines is an intrinsic motivation of the user - will raise the issue back to the humans conscious attention and thus trigger cognitive interventions that align the pattern of action to enhanced addressing of the objective function, thus offering a scaffold for learning a new behavior pattern. With continued exposition to the feedback, the pattern might solidify and become automatic even if the feedback would then be removed. Thus, for the EcoSonic system it would be interesting to evaluate possible retention effects to elucidate these mechanisms.

From this reflection we see much potential of EcoSonic to be generalized towards sonification-based habit changing technology, and its deeper understanding in various other application domains. As related work we draw the readers attention to our InfoDrops system, which enhances awareness of energy consumption while taking a shower [26]. 


\section{Conclusion and Future Work}

With our EcoSonic system we have demonstrated two novel approaches to represent consumption-specific information in an unobtrusive way using real-time sonification. The sonification methods were designed to deliver sounds that combine well with realistic (engine) sounds in the vehicle, yet create particular awareness of fuel consumption. In summary, the discussed study allows us to support most of our hypotheses listed in Section 6.1. Notably, both sonification types reduce fuel consumption significantly.

In order to confirm the results of the current study, we are currently developing a simulator which strives to reproduce the experience of car driving as closely as possible by providing an interactive view from within a car through its windshield with the help of an Oculus Rift ${ }^{8}$.

Furthermore, we plan to conduct a longitudinal study in a real-world car setting: Although this will obviously lead to a less accurate reproducibility of conditions, such a study would be even better able to capture the feasibility of our designs in a real-world context. The extended time of exposure will also allow us to observe the users' habituation to the system: Based on research on skill acquisition and automatization [27], we can not only expect a further improvement of fuel efficiency, but also an even decreased need to attend the auditory display. Thus, it might be interesting to evaluate, to which extent drivers can incorporate a fuel economic driving style even without support from a fuel efficiency display after a sufficiently long exposure to our system.

\section{Acknowledgments}

This research was supported by the Cluster of Excellence Cognitive Interaction Technology 'CITEC' (EXC 277) at Bielefeld University, which is funded by the German Research Foundation (DFG).

[1] Transportation Sector Emissions in the United States, http://www.epa.gov/climatechange/ghgemissions/sources/ transportation.html, [last accessed: 19.08.2015].

[2] J. N. Barkenbus, Eco-driving: An overlooked climate change initiative, Energy Policy 38 (2) (2010) 762-769.

\footnotetext{
${ }^{8}$ Oculus Rift: A Head-Mounted Virtual-Reality Display (https://www.oculus.com/ en-us/rift/)
} 
[3] J. J. Romm, A. A. Frank, Hybrid vehicles gain traction, Scientific American 294 (4) (2006) 72-79.

[4] M. S. Young, S. A. Birrell, N. A. Stanton, Safe driving in a green world: a review of driver performance benchmarks and technologies to support 'smart' driving., Applied ergonomics 42 (4) (2011) 533-9.

[5] L. Evans, Driver behavior effects on fuel consumption in urban driving, The Journal of the Human Factors and Ergonomics Society 21 (4) (1979) 389-398.

[6] M. Manser, M. Rakauskas, J. Graving, J. Jenness, Fuel economy driver interfaces: Develop interface recommendation. report on task 3, Fuel economy driver interfaces.

[7] C. A. Brooks, A. Rakotonirainy, In-vehicle Technologies, Advanced Driver Assistance Systems and Driver Distraction: Research challenges, in: International Conference on Driver Distraction, 2005.

[8] B. S. Jensen, M. B. Skov, N. Thiruravichandran, Studying driver attention and behaviour for three configurations of gps navigation in real traffic driving, CHI '10.

[9] M. Jeon, B. K. Davison, M. A. Nees, J. Wilson, B. N. Walker, Enhanced auditory menu cues improve dual task performance and are preferred with in-vehicle technologies, in: AutomotiveUI '09, ACM Press, p. 91.

[10] C. D. Wickens, Multiple resources and performance prediction, Theoretical issues in ergonomics science 3 (2) (2002) 159-177.

[11] M. Jeon, J. Hammerschmidt, T. Hermann, P. Bazilinskyy, S. Landry, K. A. E. Wolf, Report on the in-vehicle auditory interactions workshop: Taxonomy, challenges, and approaches, Automotive'UI 15.

[12] J. Gonder, M. Earleywine, W. Sparks, Analyzing vehicle fuel saving opportunities through intelligent driver feedback, Tech. rep., National Renewable Energy Laboratory (NREL), Golden, CO. (2012).

[13] M. J. Sullman, L. Dorn, P. Niemi, Eco-driving training of professional bus drivers-does it work?, Transportation Research Part C: Emerging Technologies 58 (2015) 749-759.

[14] J. Hammerschmidt, R. Tünnermann, T. Hermann, EcoSonic: Towards an Auditory Display Supporting a Fuel-Efficient Driving Style, in: Proceedings of SoniHED 2014, York, England, 2014, p. 56. 
[15] J. Tulusan, L. Soi, J. Paefgen, M. Brogle, T. Staake, Which ecofeedback types prefer drivers most ? (2011) 1-8.

[16] A. H. Jamson, D. L. Hibberd, N. Merat, Interface design considerations for an in-vehicle eco-driving assistance system, Transportation Research Part C: Emerging Technologies 58 (2015) 642-656.

[17] E. Hellier, A. Naweed, G. Walker, P. Husband, J. Edworthy, The influence of auditory feedback on speed choice, violations and comfort in a driving simulation game, Transportation Research Part F: Traffic Psychology and Behaviour 14 (6) (2011) 591-599.

[18] T. M. Gable, B. N. Walker, H. R. Moses, R. D. Chitloor, Advanced auditory cues on mobile phones help keep drivers' eyes on the road, in: Proceedings of AutomotiveUI '13, ACM Press, 2013, pp. 66-73.

[19] N. Powell, J. Lumsden, Exploring novel auditory displays for supporting accelerated skills acquisition and enhanced performance in motorsport, in: Proceedings of ICAD 2015.

[20] P. Larsson, D. Västfjäll, Emotional and behavioural responses to auditory interfaces in commercial vehicles, International Journal of Vehicle Noise and Vibration.

[21] R. Tünnermann, J. Hammerschmidt, T. Hermann, Blended Sonification: Sonification for Casual Interaction, in: The 19th International Conference on Auditory Display (ICAD-2013), 2013.

[22] A. Gustafsson, C. Katzeff, M. Bang, Evaluation of a pervasive game for domestic energy engagement among teenagers, Computers in Entertainment 7 (4) (2009) 1.

[23] J. Hammerschmidt, R. Tünnermann, T. Hermann, EcoSonic: Auditory Displays supporting Fuel-Efficient Driving, NordiCHI '14.

[24] Engine's Torque Map, http://f1framework.blogspot.de/2013/03/ f1-engine-maps_28.html, used with permission from the author.

[25] Brake specific fuel consumption, https://en.wikipedia.org/wiki/ Brake_specific_fuel_consumption, released under the CC BY-SA 3.0 (http://creativecommons.org/licenses/by-sa/3.0/).

[26] J. Hammerschmidt, R. Tünnermann, T. Hermann, InfoDrops: Sonification for Enhanced Awareness of Resource Consumption in the Shower, in: Proceedings of ICAD 2013. 
[27] G. D. Logan, Toward an instance theory of automatization., Psychological review 95 (4) (1988) 492. 